\title{
Factores psicosociales asociados a la realización de la prueba diagnóstica del VIH en estudiantes universitarios colombianos
}

\author{
Juan P. Sanabria-Mazo; Paula Andrea Hoyos-Hernández; Fabián Bravo
}

How to cite this article:

Sanabria-Mazo, J.P., Hoyos-Hernández, P.A. \& Bravo, F. (2020). Psychosocial factors associated with HIV testing in Colombian university students. Acta Colombiana de Psicología, 23(1), 147-157. doi: http://www.doi.org/10.14718/ ACP.2020.23.1.8

Recibido, junio 20/2018; Concepto de evaluación, enero 18/2019; Aceptado, marzo 11/2019

\author{
Juan P. Sanabria-Mazo* \\ Pontificia Universidad Javeriana, Cali, Colombia \\ ORCID: https://orcid.org/0000-0003-1688-435X \\ Paula Andrea Hoyos-Hernández \\ Pontificia Universidad Javeriana, Cali, Colombia \\ ORCID: https://orcid.org/0000-0001-7948-6338 \\ Fabián Bravo \\ Pontificia Universidad Javeriana, Cali, Colombia \\ ORCID: https://orcid.org/0000-0002-1455-1554
}

\begin{abstract}
Resumen
Los objetivos de esta investigación fueron: (a) relacionar el riesgo sexual, los conocimientos y las actitudes hacia la prueba del VIH con su realización en estudiantes universitarios colombianos; y (b) describir las barreras percibidas en aquellos que no sela habían realizado, identificando las diferencias según el sexo. Se llevó a cabo un estudio transversal en el que participaron 1057 estudiantes colombianos, y en el que se utilizó el Cuestionario Confidencial sobre Vida Sexual Activa (CCVSA), el test Barreras Hacia la Prueba del VIH, la Escala de Actitudes hacia el VIH-Sida y un cuestionario ad hoc de conocimientos sobre la prueba. Los estudiantes que se realizaron la prueba $(n=214)$ reportaron una edad inferior de iniciación sexual $(p<.01)$, un número superior de parejas sexuales $(p=.01)$ y un menor uso de condón en la primera $(p=.02)$ y la última $(p=.04)$ relación sexual, así como mayores conocimientos sobre la prueba del VIH $(p<.001)$. Las principales barreras estuvieron relacionadas con la baja percepción del riesgo de la enfermedad (29.8\%), la confianza en las parejas sexuales $(29.2 \%)$ y la falta de ofrecimiento de la prueba (25.2\%). En conclusión, la realización de la prueba está relacionada con la exposición a conductas sexuales de riesgo y con los conocimientos, mas no con las actitudes hacia la prueba. La identificación de los factores psicosociales vinculados podría contribuir al diseño de intervenciones orientadas a la prevención y al tratamiento oportuno de la enfermedad.

Palabras clave: VIH, prueba del VIH, tamizaje, estudiantes, barreras.
\end{abstract}

* Facultad de Humanidades y Ciencias Sociales, calle 18 n. ${ }^{\circ}$ 118-250, Cali, Colombia. juan.sanabria@javerianacali.edu.co 


\title{
Psychosocial factors associated with HIV testing in Colombian university students
}

\begin{abstract}
The objectives of this research were: (a) to relate sexual risk, knowledge, and attitudes towards HIV testing in Colombian university students; and (b) to describe the perceived barriers in those who had not been tested, analyzing the differences according to sex. A cross-sectional study was carried out, in which 1057 Colombian university students participated. The instruments applied were the Cuestionario Confidencial Vida Sexual Activa (CCVSA, for its Spanish acronym), the tests Barreras Hacia la Prueba del VIH, Escala de Actitudes hacia el VIH-Sida and an ad hoc questionnaire on knowledge about the test. The students who were tested $(n=214)$ reported a lower age of sexual initiation $(p<.01)$, a higher number of sexual partners $(p$ $=.01)$, and a lower use of condoms in the first $(p=.02)$ and last $(p=.04)$ sexual intercourse. They also reported more knowledge about HIV testing $(p<.001)$. The main barriers were associated with low risk perception of the disease $(29.8 \%)$, trust in sexual partners $(29.2 \%)$, and lack of offer of the test $(25.2 \%)$. In conclusion, being tested for HIV is related to exposure to risky sexual behaviors and to knowledge about the test, but not to attitudes towards it. The identification of the associated psychosocial factors could contribute to the design of interventions aimed at the prevention and timely treatment of the disease. Key words: HIV, HIV testing, screening, students, barriers.
\end{abstract}

\section{Introducción}

En la actualidad, el diagnóstico tardío del virus de la inmunodeficiencia humana (VIH) se constituye como uno de los principales problemas de salud pública en el mundo (Becky et al., 2015; Black et al., 2014; Fuster, Molero, Nouvilas, Arazo \& Dalmau, 2014). En los últimos años, esta situación se ha relacionado directamente con factores psicosociales asociados a la baja realización de la prueba diagnóstica del VIH (Lopera, Martínez \& Ray, 2011). Diversos estudios confirman que el prolongado tiempo de espera entre el momento de la infección y el inicio del tratamiento antirretroviral repercute en el aumento de nuevos casos de transmisión, en la progresión de la infección, en las dificultades para la recuperación inmunológica, en la disminución de la esperanza de vida de las personas y en el incremento de costos económicos para la sociedad (Arrivillaga et al., 2012; Bolsewicza, Vallelyb, Debattistad, Whittakere \& Fitzgeralda, 2015; Castel et al., 2012; Dai et al., 2015; Kall, Smith \& Delpech, 2012; Schnall, Rojas \& Travers, 2015).

En particular, en Colombia, de acuerdo con un informe del Ministerio de Salud y Protección Social (2015), de las 135.000 personas que fueron diagnosticadas con VIH/Sida en 2014, tan solo el $40 \%$ accedieron a un diagnóstico oportuno y recibieron tratamiento antirretroviral, siendo el $22 \%$ de los afectados jóvenes entre los 15 y 24 años. Según la Encuesta Nacional de Demografía y Salud (ENDS, 2015), cerca del $75 \%$ de los colombianos entre los 13 y los 24 años nunca se ha realizado la prueba del VIH, y aunque el $65 \%$ conoce los lugares de acceso a la prueba, solo el $25 \%$ se la ha realizado alguna vez en su vida y el $8 \%$ en los últimos 12 meses. En general, únicamente el $20 \%$ de este grupo poblacional conoce su estado serológico, circunstancia que impide identificar el alcance de la epidemia y obstaculiza el inicio temprano de los tratamientos farmacológicos y no farmacológicos.

La baja percepción de riesgo, el desconocimiento de la enfermedad y el exceso de confianza en los compañeros sexuales son los principales factores de riesgo que diferentes estudios han asociado a la adquisición del VIH en esta población (Belza et al., 2014; Fuster et al., 2014; Hoyos et al., 2019; Morales, Espada \& Orgilés, 2016; Peralta, Deeds, Hipszer \& Ghalib, 2007). Además, se ha identificado que el estigma social vinculado al VIH incrementa las probabilidades de que los jóvenes con una alta percepción de riesgo desarrollen una mayor cantidad de conductas de evitación hacia la prueba (Decker et al., 2015; Espada, Escribano, Morales \& Origlés, 2017; Fuster et al., 2014). Al respecto, García, Reyes y Caylà (2012) encontraron que la alta percepción de riesgo y el ofrecimiento de la prueba durante el embarazo fomentan que las mujeres se la realicen con más frecuencia que los hombres.

Los motivos por los que los jóvenes reportan una baja percepción de riesgo están relacionados con el exceso de confianza, la falta de conocimiento sobre la prueba diagnóstica, la concepción estereotipada de la enfermedad y el bajo número de relaciones sexuales sin protección. En cambio, la razón por la que presentan una alta percepción de riesgo está asociada a relaciones sexuales de riesgo con 
personas que sospechan que podrían tener VIH (Fuster et al., 2014). Otros estudios demuestran que, aunque la alta percepción de riesgo reduce los comportamientos de exposición a la enfermedad, esta no se relaciona con una mayor frecuencia de aplicación de la prueba (Bradley, Tsui, Kidanu \& Gillespie, 2011; Brooks, Lee, Stover \& Barkley, 2011). Del mismo modo, se ha encontrado que la actitud positiva hacia el uso de la prueba tampoco implica una mayor realización (Espada, Escribano, Orgilés, Morales \& Guillén, 2015; Morales et al., 2016).

Asimismo, investigaciones previas orientadas desde el modelo de creencias de salud de Janz y Becker (1984) han identificado que las principales barreras de los jóvenes para no realizarse la prueba son: (a) a nivel interpersonal, el desconocimiento sobre el VIH, el rechazo a obtener un diagnóstico positivo, las percepciones estigmatizadas de la enfermedad, el miedo a la divulgación de los resultados, la baja percepción de riesgo y la falta de familiarización con la prueba (Bilardi et al., 2013; Deblonde et al., 2010; Fuster et al., 2014; Kaai, Bullock, Burchell \& Major, 2012); (b) a nivel intrapersonal, la percepción negativa de los pacientes sobre la calidad de los servicios, la resistencia de los proveedores a ofrecer la prueba del VIH y el miedo al impacto del diagnóstico en su situación laboral (Becky et al., 2015; Hampanda, Ybarra \& Bull, 2014; Hoyos et al., 2012, 2013; Hyden, Allegrante \& Cohall, 2014); y (c) a nivel extrapersonal, las características sociodemográficas, las políticas públicas, las dificultades de acceso a la prueba en los sistemas de salud, la escasez de financiación y la falta de formación de recursos humanos en los profesionales de la salud (Bilardi et al., 2013; Deblonde et al., 2010; Kaai et al., 2012; Kurth, Lally, Choko, Inwani \& Fortenberry, 2015; Newman et al., 2013).

En particular, las barreras intrapersonales, como las habilidades y las motivaciones, están conectadas con las extrapersonales, como los elementos sociales, culturales, económicos y políticos (Begley, McLaws, Ross \& Gold, 2008). En este sentido, las limitaciones estructurales dificultan la accesibilidad a los servicios y tienen un impacto negativo en las motivaciones de los jóvenes para realizarse la prueba (Deblonde et al., 2010; Newman et al., 2013), mientras que las percepciones de las políticas públicas de cada país sobre la enfermedad pueden perpetuar el estigma hacia el VIH, situación que impacta directamente en la actitud de la comunidad. De igual forma, el miedo a la interacción con los pacientes, la falta de comprensión de la epidemiología del VIH por el desconocimiento de los protocolos de las pruebas por parte de los profesionales de la salud pueden convertirse en barreras (Kaai et al., 2012; Kall et al., 2012; Deblonde et al., 2010).
Por otra parte, el desconocimiento del estado serológico imposibilita el inicio oportuno de los tratamientos antirretrovirales y dificulta que los jóvenes desarrollen comportamientos de protección (Becky et al., 2015; Cooney, Hiransuthikul \& Lertmaharit, 2015; Deblonde et al., 2010; Flowers, Knussen, Li \& McDaid, 2013; Hoyos et al., 2013; Kall et al., 2012; Schnall et al., 2015). La realización de la prueba diagnóstica del VIH se relaciona, de acuerdo con el modelo de creencias en salud (Janz \& Becker, 1984), con los conocimientos, las actitudes y la autoeficacia de las personas, así como con la percepción de las barreras y los facilitadores asociados a este proceso. En este contexto, la identificación de los múltiples factores psicosociales asociados a su baja realización en Colombia podría contribuir al diseño de campañas orientadas a la prevención y al tratamiento oportuno de la enfermedad que estén orientados a la reducción de los índices de mortalidad y morbilidad reportados (Fuster et al., 2014; Hoyos et al., 2013; Newman et al., 2013). Además, podría servir para que los servicios de salud sexual y salud reproductiva respondan a las necesidades particulares de este grupo poblacional.

Por este motivo, la presente investigación tuvo dos objetivos principales: (a) relacionar el riesgo sexual, los conocimientos y las actitudes hacia la prueba del VIH con su realización en estudiantes universitarios colombianos; y (b) describir las barreras percibidas en aquellos que no se la habían realizado, identificando las diferencias según el sexo.

\section{Método}

\section{Tipo de estudio}

Se realizó un estudio transversal, de carácter descriptivocorrelacional (Hernández, Fernández \& Baptista, 2010).

\section{Participantes}

Participaron 1057 estudiantes de una universidad de Cali, Colombia, seleccionados de manera intencional. Los criterios de inclusión fueron que tuvieran entre 18 y 22 años, que estuvieran matriculados en la institución educativa y que no presentaran alteraciones cognitivas.

\section{Instrumentos}

Cuestionario de información general.Se elaboró un cuestionario para caracterizar los datos sociodemográficos, como la edad, el sexo, la orientación sexual, el nivel socioeconómico, los ingresos familiares, el año académico, la religión y las personas con las que viven. 
150

Cuestionario Confidencial sobre Vida Sexual Activa (CCVSA). Este instrumento, diseñado por el Ministerio de Salud de Colombia (1997), se empleó para identificar las prácticas sexuales, el uso del preservativo y el número de parejas sexuales. El formato de respuesta es dicotómico ("sí" o "no").

Barreras hacia la Prueba del VIH. Este instrumento, diseñado por Peralta et al. (2007), cuenta originalmente con un listado de 16 barreras clasificadas en seis bloques. De este listado, los participantes deben señalar todas las razones por las que no se han realizado la prueba diagnóstica del VIH. Este cuestionario fue validado en jóvenes estadounidenses (Peralta et al., 2007) y españoles (Morales et al., 2016) entre los 14 y los 24 años, y cuenta con un alfa de Cronbach de .71. La fiabilidad identificada en esta investigación fue consistente con la reportada por Peralta et al. (2007) y Morales et al. (2016). En este estudio se incluyó un listado adicional para describir las fuentes de información sobre la prueba y la frecuencia de aplicación.

Conocimientos asociados a la prueba diagnóstica del VIH. Este instrumento, diseñado por los investigadores de este estudio, consta de 14 ítems y tiene como objetivo medir los conocimientos correctos (siete ítems) e incorrectos (siete ítems) sobre la prueba del VIH. El formato de respuesta es de "verdadero" (acierto) y "falso/no sé" (desacierto), y las puntuaciones totales oscilan en un rango de 0 a 14, donde los puntajes más altos de acierto indican un mayor conocimiento sobre la prueba del VIH. En el proceso de evaluación de contenido del instrumento se contó con el apoyo de jueces expertos externos, quienes se encargaron de evaluar la coherencia, pertinencia y claridad de los ítems. Se solicitó a un grupo de 30 participantes que cumplían con los criterios de inclusión de la muestra que respondieran el cuestionario para verificar la adecuada comprensión de las preguntas. El alfa de Cronbach reportado en este instrumento fue de .73 .

Escala de Actitudes hacia el VIH-Sida. Este instrumento, diseñado por Espada, Ballester, Huedo, Secades y Martínez (2013), cuenta con un total de 12 ítems y tiene como objetivo evaluar las actitudes sobre el VIH/Sida. Para efectos de este estudio, se empleó únicamente la subescala de actitud hacia la prueba del VIH. Esta subescala cuenta con un total de dos ítems con un formato de respuesta tipo Likert, en el que 1 corresponde a "totalmente en desacuerdo" y 4 a "totalmente de acuerdo"; y las puntuaciones totales oscilan entre 2 y 8 , donde los puntajes más altos indican mayor favorabilidad hacia la prueba del VIH. Esta escala fue validada en jóvenes españoles, donde se reporta un alfa de Cronbach total de .81. En esta investigación el alfa reportado fue de .82 .

\section{Procedimiento}

Inicialmente, se contactó a los directivos de la universidad para presentar la propuesta y solicitar la autorización para acceder a la población de estudio. Después, se invitó a participar a todos los estudiantes que cumplieran con los criterios de inclusión; una vez conocieron y aceptaron los alcances del estudio, se les solicitó que firmaran el consentimiento informado. La aplicación de la batería de instrumentos se realizó en salones de la institución adecuados exclusivamente para esta actividad. El tiempo de diligenciamiento de los instrumentos fue de, aproximadamente, 25 minutos, y la aplicación estuvo a cargo del equipo de investigación, con el apoyo de estudiantes de pregrado y posgrado capacitados para este proceso, en los meses de julio y agosto de 2017.

Esta investigación estuvo orientada por la resolución n. ${ }^{\circ} 008430$ de 1993 del Ministerio de Salud, en la cual se exponen las normas científicas, técnicas y administrativas para la investigación en salud con seres humanos. Además, se tuvieron en cuenta las consideraciones éticas definidas en el Manual Deontológico y Bioético de Psicología y la ley 1090 del 2006 que regula el ejercicio de la Psicología en Colombia.

\section{Análisis de datos}

Las características sociodemográficas de los participantes se analizaron, según el tipo de variable, mediante promedios o porcentajes. Para comparar las prácticas sexuales, los conocimientos, las actitudes y las barreras entre los participantes que se realizaron o no la prueba, o por sexo, se utilizaron pruebas $T$ para muestras independientes y análisis de prueba $X^{2}$. Antes de aplicar la prueba T, se realizó una revisión del cumplimiento de los requisitos de normalidad - mediante la prueba de Kolmogorov Smirnov-y de la homogeneidad de variancias - mediante la prueba de Levene- Los resultados obtenidos permitieron aceptar el supuesto de normalidad en la distribución de las puntuaciones de las variables en los grupos de estudio. El supuesto de igualdad o de diferencia de variancias se asumió en las asociaciones de las variables, considerando los resultados obtenidos en la prueba de Levene. Finalmente, se calculó el coeficiente de phi $(\varphi)$ y el tamaño del efecto (Cohen, 1988) cuando las asociaciones y las diferencias fueron estadísticamente significativas. Finalmente, el tamaño del efecto se clasificó según la propuesta original de Cohen: grande $(d>0.8)$, 
medio $(0.8>d>0.5)$ y pequeño $(d<0.3)$. Los datos se analizaron mediante el software SPSS, versión 23.

\section{Resultados}

En este apartado se presentan los factores psicosociales asociados a la realización de la prueba diagnóstica del VIH en los estudiantes universitarios colombianos.

\section{Datos sociodemográficos}

En total, participaron 1057 estudiantes, 651 mujeres (63\%) y 384 hombres (37\%), con edades entre los 18 y los 22 años $(M=19.59, D E=1.27)$, que pertenecían al primer $(23.1 \%)$, segundo (29\%), tercer (28.8\%), cuarto $(14.6 \%)$ y quinto ( $4.5 \%)$ año de una universidad de Cali, Colombia. De ellos, 214 (20.2 \%) reportaron haberse realizado la prueba del VIH.

En la Tabla 1 se presentan los datos sociodemográficos de los participantes, diferenciados según aquellos que se han realizado o no la prueba.

Como se puede observar, no se encontraron diferencias significativas entre los participantes que reportaron haberse realizado o no la prueba diagnóstica del VIH en las variables sexo $\left(X^{2}=0.011, p=.91\right)$, estatus socioeconómico $\left(X^{2}=0.002, p=.96\right)$, ingresos familiares $\left(X^{2}=0.059\right.$, $p=.80)$ y personas con quienes viven $\left(X^{2}=3.293, p=.07\right)$.
No obstante, se identificaron asociaciones significativas de baja magnitud según la orientación sexual y la práctica religiosa, con mayor presencia de participantes de orientación homosexual o bisexual $\left(X^{2}=16.482, p<.001, \varphi=0.12\right)$, y menor de practicantes de alguna religión $\left(X^{2}=7.311, p<.01\right.$, $\varphi=0.08)$, en el grupo que se realizó la prueba diagnóstica.

\section{Prácticas sexuales y prueba del VIH}

Con el propósito de relacionar las prácticas sexuales con la prueba, se compararon los dos grupos con respecto a la edad de iniciación sexual, los tipos de relaciones sexuales, la cantidad de parejas sexuales y el uso del condón durante la primera y última relación sexual, limitando la muestra al $83.2 \%$ que afirmó haber mantenido en su vida algún tipo de relación sexual vaginal, anal o bucogenital. En la Tabla 2 se aprecia que el porcentaje que reportó haber tenido relaciones sexuales vaginales fue significativamente superior $\left(X^{2}=29.38, p<.001, \varphi=0.16\right)$ en los participantes que se realizaron la prueba del VIH (93.7 \%) que en los que no (77\%). Esta asociación, no obstante, fue de baja magnitud.

Además, se identificaron diferencias estadísticamente significativas asociadas a la edad de la primera relación sexual $(t=-3.01, p<.01)$ y el número de parejas sexuales en los últimos seis meses $(t=1.99, p=.04)$, siendo ligeramente inferior la edad de iniciación sexual (diferencia de medias $=-0.45, d=0.23)$ y ligeramente superior el número

Tabla 1.

Datos sociodemográficos, diferenciados por los participantes que se han realizado o no la prueba diagnóstica del VIH

\begin{tabular}{|c|c|c|c|c|c|c|c|c|}
\hline \multirow{2}{*}{ Variable } & \multicolumn{2}{|c|}{ Sí prueba } & \multicolumn{2}{|c|}{ No prueba } & \multicolumn{2}{|c|}{ Total } & \multirow{2}{*}{$X^{2}$} & \multirow{2}{*}{$p$} \\
\hline & $n$ & $\%$ & $n$ & $\%$ & $n$ & $\%$ & & \\
\hline Sexo & & & & & & & 0.011 & .91 \\
\hline Hombres & 77 & 36.7 & 305 & 37.1 & 382 & 37 & & \\
\hline Mujeres & 133 & 63.3 & 518 & 62.9 & 651 & 63 & & \\
\hline Estatus socioeconómico & & & & & & & 0.002 & .96 \\
\hline Bajo & 19 & 8.9 & 74 & 8.8 & 93 & 8.8 & & \\
\hline Medio/alto & 195 & 91.1 & 769 & 91.2 & 964 & 91.2 & & \\
\hline Ingresos familiares & & & & & & & 0.059 & .80 \\
\hline Inferiores a 2 SMMLV & 16 & 7.5 & 59 & 7 & 75 & 7.1 & & \\
\hline Superiores a 2 SMMLV & 198 & 92.5 & 784 & 93 & 982 & 92.9 & & \\
\hline Vive con & & & & & & & 3.293 & .07 \\
\hline Padres & 143 & 66.8 & 616 & 73.1 & 759 & 71.8 & & \\
\hline Otros & 71 & 33.2 & 227 & 26.9 & 298 & 28.2 & & \\
\hline Orientación sexual & & & & & & & 16.482 & $.001 * * *$ \\
\hline Heterosexual & 175 & 81.8 & 770 & 91.3 & 945 & 89.4 & & \\
\hline Homosexual/bisexual & 39 & 18.2 & 73 & 8.7 & 112 & 10.6 & & \\
\hline Practicante religioso & & & & & & & 7.311 & $.007^{* *}$ \\
\hline Sí & 85 & 39.7 & 422 & 50.1 & 507 & 48 & & \\
\hline No & 129 & 60.3 & 421 & 49.9 & 550 & 52 & & \\
\hline
\end{tabular}

Nota. $* \mathrm{p}<.05, * * \mathrm{p}<.01,{ }^{* * *} \mathrm{p}<.001$. SMMLV: Salario mínimo mensual legal vigente. 
Tabla 2.

Prácticas sexuales, diferenciadas por los participantes que se han realizado o no la prueba del VIH

\begin{tabular}{|c|c|c|c|c|c|c|c|c|}
\hline \multirow{2}{*}{ Variable } & \multicolumn{2}{|c|}{ Total } & \multicolumn{2}{|c|}{ Sí prueba } & \multicolumn{2}{|c|}{ No prueba } & \multirow{2}{*}{$X^{2}$} & \multirow{2}{*}{$p$} \\
\hline & $n$ & $\%$ & $n$ & $\%$ & $n$ & $\%$ & & \\
\hline Relaciones sexuales vaginales & 829 & 80.3 & 194 & 93.7 & 635 & 77 & 29.38 & $.001 * * *$ \\
\hline Uso del condón primera relación & 628 & 76.7 & 135 & 71.4 & 493 & 78.5 & 5.01 & $.025^{*}$ \\
\hline \multirow[t]{2}{*}{ Uso del condón última relación } & 415 & 49.8 & 85 & 43.6 & 330 & 51.7 & 3.95 & $.047 *$ \\
\hline & $M$ & $D E$ & $M$ & $D E$ & $M$ & $D E$ & $t$ & $P$ \\
\hline Edad primera relación & 16.2 & 1.8 & 15.93 & 2.02 & 16.38 & 1.82 & -3.01 & $.003 * *$ \\
\hline Parejas sexuales últimos 6 meses & 1.64 & 1.32 & 1.85 & 1.79 & 1.58 & 1.13 & 1.99 & $.048^{*}$ \\
\hline
\end{tabular}

Nota. $* \mathrm{p}<.05, * * \mathrm{p}<.01, * * * \mathrm{p}<.001$.

de parejas sexuales (diferencia de medias $=0.27, d=0.2$ ) en los participantes que se realizaron la prueba. El tamaño de efecto entre ambas diferencias de medias fue pequeño.

En cuanto al uso del condón, se encontraron asociaciones significativas de baja magnitud en la primera $\left(X^{2}=5.01\right.$, $p=.02, \varphi=0.07)$ y la última $\left(X^{2}=3.95, p=.04, \varphi=0.06\right)$ relación sexual, evidenciándose un mayor porcentaje de uso en el primer (78.5\% vs. $71.4 \%$ ) y el último encuentro sexual (51.7 \% vs. $43.6 \%)$ en los participantes que no se la realizaron.

\section{Conocimientos asociados a la prueba del VIH}

Para la muestra total, las principales fuentes de información sobre la prueba del VIH fueron los profesionales de la salud (37.1\%), el internet (29.5\%) y las instituciones educativas $(26.3 \%)$. Por su parte, los programas de TV o de radio (19.3\%), los artículos en prensa $(19.1 \%)$ y la publicidad (5.2\%) fueron los medios menos empleados.
Para medir los conocimientos, se aplicaron 14 ítems compuestos por afirmaciones correctas e incorrectas. Los participantes que se realizaron la prueba presentaron puntuaciones (en un rango de 0 a 14) más altas $(M=9.21$, $D E=1.98)$ en comparación con los que no se la realizaron $(M=7.87, D E=2.19)$, siendo estas diferencias significativas $(t=7.79, p<.001, d=0.65)$, con un tamaño de efecto medio. Se destaca, además, que al comparar por sexo no se evidenciaron diferencias significativas $(t=1.69, p=.09)$ en los conocimientos reportados por hombres $(M=8.30$, $D E=2.46)$ y mujeres $(M=8.03, D E=2.06)$.

Con respecto a los conocimientos incorrectos, se encontró que los participantes que aseguraron haberse realizado la prueba del VIH presentaron índices de acierto más altos en las afirmaciones "si los resultados de la prueba de mi pareja son negativos, no es necesario hacerme la prueba" (90.1\% vs. $80.1 \%$ ) y "si los resultados de mi prueba salen negativos ya no tengo de qué preocuparme" ( $66 \%$ vs. $51 \%$ ), siendo

Tabla 3.

Aciertos en conocimientos incorrectos sobre la prueba del VIH, diferenciados por los participantes que se la han realizado y los que no

\begin{tabular}{|c|c|c|c|c|c|c|c|c|}
\hline \multirow[t]{2}{*}{ Conocimientos incorrectos } & \multicolumn{2}{|c|}{$\begin{array}{l}\text { Total de } \\
\text { aciertos }\end{array}$} & \multicolumn{2}{|c|}{$\begin{array}{l}\text { Sí prueba } \\
\text { aciertos }\end{array}$} & \multicolumn{2}{|c|}{$\begin{array}{l}\text { No prueba } \\
\text { aciertos }\end{array}$} & \multirow[t]{2}{*}{$X^{2}$} & \multirow[t]{2}{*}{$p$} \\
\hline & $n$ & $\%$ & $n$ & $\%$ & $n$ & $\%$ & & \\
\hline $\begin{array}{l}\text { 1. Si soy una persona de orientación heterosexual, no necesito real- } \\
\text { izarme la prueba del VIH. }\end{array}$ & 814 & 93.6 & 190 & 96 & 624 & 92.9 & 2.444 & .118 \\
\hline $\begin{array}{l}\text { 2. Las personas que han tenido relaciones sexuales solo con una } \\
\text { pareja no necesitan realizarse la prueba. }\end{array}$ & 793 & 90.5 & 189 & 93.6 & 604 & 89.6 & 2.827 & .093 \\
\hline $\begin{array}{l}\text { 3. Si los resultados de la prueba de mi pareja son negativos, no es } \\
\text { necesario hacerme la prueba. }\end{array}$ & 719 & 82.5 & 182 & 90.1 & 537 & 80.1 & 10.62 & $.001 * * *$ \\
\hline $\begin{array}{l}\text { 4. Únicamente las personas mayores de edad pueden realizarse la } \\
\text { prueba del VIH. }\end{array}$ & 506 & 57.8 & 129 & 63.9 & 377 & 55.9 & 4.003 & $.045^{*}$ \\
\hline $\begin{array}{l}\text { 5. Si los resultados de mi prueba salen negativos, ya no tengo de qué } \\
\text { preocuparme. }\end{array}$ & 479 & 55.1 & 132 & 66 & 347 & 51.8 & 12.56 & $.001 * * *$ \\
\hline 6. Las pruebas rápidas de detección del VIH no son fiables. & 226 & 25.9 & 68 & 33.7 & 158 & 23.5 & 8.348 & $.004 * *$ \\
\hline 7. Solo puedo saber que tengo VIH mediante una prueba en sangre. & 119 & 13.6 & 26 & 12.9 & 93 & 13.8 & 0.103 & .749 \\
\hline
\end{tabular}

Nota. $* \mathrm{p}<.05, * * \mathrm{p}<.01, * * * \mathrm{p}<.001$. 
Tabla 4.

Aciertos en conocimientos correctos sobre la prueba del VIH, diferenciados por los participantes que se la han realizado y los que no

\begin{tabular}{|c|c|c|c|c|c|c|c|c|}
\hline \multirow[t]{2}{*}{ Conocimientos correctos } & \multicolumn{2}{|c|}{ Total de aciertos } & \multicolumn{2}{|c|}{$\begin{array}{l}\text { Sí prueba } \\
\text { aciertos }\end{array}$} & \multicolumn{2}{|c|}{$\begin{array}{l}\text { No prueba } \\
\text { aciertos }\end{array}$} & \multirow[t]{2}{*}{$X^{2}$} & \multirow[t]{2}{*}{$p$} \\
\hline & $n$ & $\%$ & $n$ & $\%$ & $n$ & $\%$ & & \\
\hline $\begin{array}{l}\text { 8. La única manera de saber con seguridad si tengo } \\
\text { VIH es mediante la prueba diagnóstica. }\end{array}$ & 708 & 80.7 & 171 & 84.7 & 537 & 79.6 & 2.597 & .107 \\
\hline $\begin{array}{l}\text { 9. Los resultados de la prueba del VIH son } \\
\text { confidenciales. }\end{array}$ & 690 & 79.3 & 173 & 86.9 & 517 & 77 & 9.140 & $.003 * *$ \\
\hline $\begin{array}{l}\text { 10. Los profesionales de la salud no pueden realizarme } \\
\text { la prueba del VIH sin mi consentimiento. }\end{array}$ & 682 & 78 & 169 & 84.1 & 513 & 76.2 & 5.569 & $.018^{*}$ \\
\hline 11. Los resultados de las pruebas del VIH son fiables. & 607 & 69.4 & 149 & 73.8 & 458 & 68.1 & 2.383 & .123 \\
\hline $\begin{array}{l}\text { 12. Puedo solicitar la prueba del VIH en mi institución } \\
\text { de salud (IPS-EPS). }\end{array}$ & 545 & 62.5 & 165 & 82.5 & 380 & 56.5 & 44.29 & $.001 * * *$ \\
\hline 13. La prueba de detección del VIH es gratuita. & 127 & 14.5 & 65 & 32.2 & 62 & 9.2 & 65.71 & $.001 * * *$ \\
\hline $\begin{array}{l}\text { 14. Solamente puedo hacerme la prueba de VIH si } \\
\text { recibo una asesoría pre y posprueba. }\end{array}$ & 110 & 12.6 & 35 & 17.4 & 75 & 11.2 & 5.456 & $.020^{*}$ \\
\hline
\end{tabular}

Nota. $* \mathrm{p}<.05, * * \mathrm{p}<.01, * * * \mathrm{p}<.001$.

todas estas diferencias significativas $(p<.05)$. Por su parte, frente a los conocimientos correctos, reportaron índices de acierto más altos en las afirmaciones "los resultados de la prueba del VIH son confidenciales" (86.9 vs. 77) y "los profesionales de la salud no pueden realizarme la prueba sin mi consentimiento" ( 84.1 vs. 76.2$)$, evidenciándose, del mismo modo, diferencias significativas $(p<.02)$.

En las tablas 3 y 4 se presentan los índices de acierto de los conocimientos sobre la prueba del VIH, diferenciados por los participantes que se la han realizado y los que no.

En los participantes de ambos grupos se resalta un bajo índice de acierto en afirmaciones incorrectas como "solo puedo saber que tengo VIH mediante una prueba en sangre" (13.6\%), "las pruebas rápidas de detección del VIH no son fiables" (25.9\%) y "únicamente las personas mayores de edad pueden realizarse la prueba" (57.8 \%), así como en afirmaciones correctas como "solamente puedo hacerme la prueba si recibo una asesoría pre y posprueba" (12.6\%), "la prueba de detección del VIH es gratuita" (14.5\%) y "puedo solicitar la prueba en mi institución de salud (IPSEPS)" (62.5\%).

\section{Actitudes hacia la prueba}

Las actitudes se evaluaron a partir de las puntuaciones de los participantes en dos ítems (en un rango de 0 a 8) asociados a las actitudes de las personas hacia la prueba diagnóstica del VIH. Aunque los que se realizaron la prueba presentaron puntuaciones más altas $(M=7.35, D E=1.32)$ en comparación con los que no $(M=7.23, D E=1.21)$, la diferencia no fue significativa $(t=1.29, p=.19)$. Sin embargo, se encontró que, al comparar por sexo, se evidenciaron diferencias significativas $(t=-2.08, p=.04, d=0.07)$ en las actitudes reportadas por hombres $(M=7.16, D E=1.29)$ y mujeres $(M=7.33, D E=1.17)$, aunque con un tamaño de efecto irrelevante.

En general, el $69 \%$ de los participantes consideró estar totalmente de acuerdo con realizarse la prueba si tuviera una práctica sexual de riesgo. Además, el $72 \%$ aseguró estar totalmente de acuerdo en recomendar a un amigo hacerse las pruebas de detección del VIH si hubiese mantenido prácticas sexuales de riesgo.

\section{Barreras hacia la prueba}

Los principales motivos por los que hombres y mujeres no se han realizado la prueba diagnóstica del VIH fueron porque están seguros de que sus parejas sexuales no tienen VIH (29.8 \%), porque confían en las parejas sexuales que han tenido $(29.2 \%)$, porque nunca les han ofrecido realizarse la prueba $(25.2 \%)$ y porque no han tenido relaciones sexuales sin protección (23.8 \%). En la Tabla 5 se presentan las barreras que asociaron los participantes a la prueba diagnóstica del VIH.

En cuanto a las diferencias por sexo, se identificó que las mujeres no se realizaron la prueba en un porcentaje más alto que los hombres, por razones como no haber tenido relaciones sexuales ( $20.7 \%$ vs. $11.3 \%$ ) o no querer que sus padres supieran que han tenido relaciones sexuales $(3.7 \%$ vs. $2.7 \%)$, siendo estas diferencias significativas $(p<.015)$. Por su parte, los hombres no se la hicieron en un porcentaje más alto que las mujeres por razones como 
Tabla 5 .

Barreras asociadas a la prueba diagnóstica del VIH

\begin{tabular}{|c|c|c|c|c|c|c|c|c|}
\hline \multirow{2}{*}{ Barreras percibidas } & \multicolumn{2}{|c|}{ Total } & \multicolumn{2}{|c|}{ Hombres } & \multicolumn{2}{|c|}{ Mujeres } & \multirow{2}{*}{$X^{2}$} & \multirow[b]{2}{*}{$p$} \\
\hline & $n$ & $\%$ & $n$ & $\%$ & $n$ & $\%$ & & \\
\hline 1. No he tenido relaciones sexuales. & 178 & 17.2 & 43 & 11.3 & 135 & 20.7 & 15.17 & $.001 * * *$ \\
\hline $\begin{array}{l}\text { 2. He tenido relaciones sexuales, pero siempre he uti- } \\
\text { lizado condón. }\end{array}$ & 246 & 23.8 & 109 & 28.5 & 137 & 21 & 7.44 & $.006 * *$ \\
\hline $\begin{array}{l}\text { 3. Pienso que el VIH solo afecta a trabajadores sexuales/ } \\
\text { homosexuales. }\end{array}$ & 5 & 0.5 & 5 & 1.3 & 0 & 0 & 8.56 & $.003 * *$ \\
\hline $\begin{array}{l}\text { 4. No quiero que mis papás sepan que he tenido relacio- } \\
\text { nes sexuales. }\end{array}$ & 28 & 2.7 & 4 & 1 & 24 & 3.7 & 6.36 & $.012 *$ \\
\hline $\begin{array}{l}\text { 5. Estoy seguro(a) de que mis parejas sexuales no tienen } \\
\text { VIH. }\end{array}$ & 308 & 29.8 & 133 & 34.8 & 175 & 26.9 & 7.24 & $.007 * *$ \\
\hline 6. Nunca me han ofrecido hacerme la prueba de VIH. & 260 & 25.2 & 102 & 26.7 & 158 & 24.3 & 0.75 & .385 \\
\hline 7. No sé dónde puedo hacerme la prueba. & 111 & 10.7 & 50 & 13.1 & 61 & 9.4 & 3.47 & .062 \\
\hline 8. Me asustaría tener un resultado positivo de VIH. & 88 & 8.5 & 46 & 12 & 42 & 6.5 & 9.65 & $.002 * *$ \\
\hline $\begin{array}{l}\text { 9. Me preocupa no saber cómo actuar si me detectan } \\
\text { VIH. }\end{array}$ & 74 & 7.2 & 32 & 8.4 & 42 & 6.5 & 1.34 & .247 \\
\hline 10. Me preocupa morirme si me diagnostican VIH. & 30 & 2.9 & 18 & 4.7 & 12 & 1.8 & 7.02 & $.008 * *$ \\
\hline 11. No quiero que nadie sepa si tengo VIH. & 10 & 1 & 7 & 1.8 & 3 & 0.5 & 4.72 & $.030 *$ \\
\hline 12. Me da miedo que me saquen sangre. & 36 & 3.5 & 12 & 3.1 & 24 & 3.7 & 0.21 & .645 \\
\hline 13. Tengo que pagar por hacerme la prueba. & 36 & 3.5 & 11 & 2.9 & 25 & 3.8 & 0.66 & .416 \\
\hline 14. No quiero esperar a recibir los resultados. & 13 & 1.3 & 6 & 1.6 & 7 & 1.1 & 0.47 & .490 \\
\hline 15. Pienso que no es posible que tenga VIH. & 230 & 22.3 & 90 & 23.6 & 140 & 21.5 & 0.58 & .443 \\
\hline 16. No sé qué beneficios tiene hacerme la prueba. & 18 & 1.7 & 6 & 1.6 & 12 & 1.8 & 0.10 & .746 \\
\hline
\end{tabular}

Nota. $* \mathrm{p}<.05, * * \mathrm{p}<.01, * * * \mathrm{p}<.001$.

estar seguros de que su pareja no tenía el virus (34.8\% vs. $26.9 \%$ ), haber tenido relaciones sexuales siempre con condón ( $28.5 \%$ vs. $21 \%$ ) y temor a obtener un resultado positivo de VIH (12\% vs. $9.65 \%)$, también con diferencias significativas $(p<.01)$.

\section{Discusión}

Los objetivos principales de esta investigación fueron relacionar el riesgo sexual, los conocimientos y las actitudes hacia la prueba del VIH con su realización en estudiantes universitarios colombianos y describir las barreras percibidas en aquellos que no se la habían realizado, identificando las diferencias según el sexo Los resultados encontrados evidencian que, aunque la mayoría ya había iniciado su vida sexual $(83.2 \%)$, cerca de la mitad no utilizó condón en su última relación sexual (49.8\%). Esta situación se ha relacionado en la literatura a factores como la falta de disponibilidad del condón en el momento de la relación sexual, la baja percepción de riesgo de adquirir el VIH y la vergüenza para exigirlo a la pareja durante los encuentros (Belza et al., 2014; Fuster et al., 2014; Hoyos et al., 2019; Morales et al., 2016). Es importante mencionar que, a pesar de que la exposición a riesgo sexual de los participantes fue alta y que la mayoría sabía de la existencia de la prueba diagnóstica del VIH, únicamente el $20 \%$ se la realizó para conocer su estado serológico, porcentaje que se aproxima al $22 \%$ que se reportó en el estudio de Hoyos et al. (2012) en España, al 29.5 \% de la investigación de Peralta et al. (2007) en Estados Unidos y al $25 \%$ del informe de la ENDS (2015) en Colombia.

Ahora bien, aunque el puntaje de conocimientos sobre la prueba que obtuvieron los participantes que se la habían realizado fue superior que los que no, y que la mayoría de la información la adquirieron de fuentes de información como los profesionales de salud, el internet y las instituciones educativas, se evidenció que una cantidad significativa desconoce que en Colombia, por ley, la prueba debe ser gratuita, que se puede solicitar siempre que se requiera en las Empresas Prestadoras de Servicios de Salud (EPS) como parte del Plan Obligatorio de Salud (POS), que es de libre acceso para personas de todas las edades y que para realizársela se debe recibir una asesoría previa en la que se explica información básica sobre la función de la prueba y una asesoría posterior en la que se ayuda a entender el resultado (Profamilia, 2017).

Igualmente, algunos de los participantes del estudio desconocían que los profesionales de la salud no pueden revelar información sobre el diagnóstico, los resultados y 
la evolución de la enfermedad (Organización Mundial de la Salud [OMS], 2012). En una alta proporción, no eran conscientes de la existencia de pruebas diferentes a las de sangre de tamizaje (ELISA y pruebas rápidas de detección) y confirmatorias (Western Blot), como las de saliva y orina, para diagnosticar la enfermedad (Ministerio de Salud y Protección Social [UNFPA], 2011). Del mismo modo, no sabían que es recomendable realizarse la prueba cada año y que, aunque los resultados sean negativos, deben seguir realizando un seguimiento a su estado serológico (Infosida, 2016).

Otro factor reportado en investigaciones anteriores es la relación entre la realización de la prueba y la actitud hacia el VIH de los participantes (Espada et al., 2015; Morales et al., 2016). A diferencia de los resultados obtenidos en estudios previos (Belza et al., 2014; Bilardi et al., 2013; Bradley et al., 2011; Brooks et al., 2011; Decker et al., 2015; Deblonde et al., 2010; Espada et al., 2017; Fuster et al., 2014; Kaai et al., 2012), en esta investigación se encontró que la exposición a conductas de riesgo sexual y los conocimientos sobre la prueba del VIH estuvieron relacionados con una mayor realización de la misma, con un efecto medio para los conocimientos y pequeño para los riesgos sexuales. No obstante, en concordancia con los hallazgos de los estudios citados, no se identificó una relación entre las actitudes favorables hacia la prueba y su realización.

Por otra parte, al igual que se ha reportado en otros estudios, estos resultados demuestran que las principales barreras personales asociadas a la baja realización de la prueba diagnóstica del VIH son el exceso de confianza en las parejas sexuales, la baja percepción de exposición de riesgo a la enfermedad y la seguridad de haber mantenido relaciones sexuales con protección (Belza et al., 2014; Fuster et al., 2014; Morales et al., 2016; Peralta et al., 2007). En este punto, se destaca que la confianza en las parejas sexuales, la seguridad de haber tenido relaciones sexuales siempre con condón y el temor a obtener resultados positivos son motivos significativamente más presentes en hombres que en mujeres. En cambio, no querer que sus padres supieran que han tenido relaciones sexuales fue un motivo más evidente en las mujeres de esta investigación.

Otros motivos relacionados fueron la falta de ofrecimiento de la prueba por parte de las entidades de salud y porque aún no habían iniciado su vida sexual (Morales et al., 2016; Peralta et al., 2007). En esta investigación, el miedo a obtener un diagnóstico positivo, las percepciones estigmatizadas de la enfermedad y el miedo a la divulgación de los resultados no fueron barreras interpersonales que interfirieron en la realización de la prueba (Bilardi et al., 2013; Deblonde et al., 2010; Kaai et al., 2012).
En la actualidad, las estrategias de intervención se han centrado en identificar las percepciones de riesgo sobre el VIH, reconocer las estrategias para mejorar la accesibilidad de las pruebas, incrementar el número de profesionales de salud especializados y reducir el estigma asociado a la enfermedad (Deblonde et al., 2010; Kaai et al., 2012; Kall et al., 2012; Newman et al., 2013). Autores como Becky et al. (2015) propusieron algunos facilitadores que incrementan las probabilidades de que las personas se realicen la prueba, entre estos se encuentran: (a) los políticos, que están asociados con la eliminación del requisito de consentimiento por escrito, la racionalización del asesoramiento previo y posterior al VIH y la obligación a los médicos de que realizaran pruebas de VIH rutinarias; (b) los comunitarios, que hacen referencia a la disminución del estigma y el desarrollo de campañas orientadas a la aceptación de los pacientes; (c) los prácticos, que implican el asesoramiento sobre el VIH, la educación a los profesionales de la salud, la inclusión de la prueba como una práctica rutinaria en el trabajo clínico y el uso de las pruebas de VIH orales; y (d) los personales, que consisten en educar a las personas sobre el riesgo de contraer el VIH, incrementar la aceptación de las ventajas de realizarse la prueba, atender oportunamente a las solicitudes de los pacientes y abordar a la sexualidad de una manera integral.

Ante este panorama, resulta prioritario fortalecer la formación conceptual y el empoderamiento de individuos, familias, comunidades e instituciones de salud respecto a las creencias y conocimientos asociados a la sexualidad y a los derechos sexuales y derechos reproductivos, desde una perspectiva del autocuidado en la que prime la información científica y en la que se establezca un enfoque diferencial ajustado al curso de vida de las personas, aportando, a su vez, a la reducción del estigma y la discriminación. Además, fortalecer los programas de educación en salud sexual y reproductiva desde la primera infancia permitirá contribuir al bienestar y la calidad de vida de las poblaciones y disminuir los casos de embarazo adolescente, el VIH y otras ITS. Ahora bien, estas intervenciones deben darse articuladas con el marco de las políticas públicas de cada país (Hoyos et al., 2019; Morales et al., 2016; Sevilla, Sanabria, Orcasita \& Palma, 2016), las cuales deben favorecer la inclusión, la atención integral y el acceso fácil a los programas de salud sexual y salud reproductiva.

Los hallazgos de esta investigación, por tanto, se presentan como una herramienta para el diseño de programas de promoción de la salud sexual y salud reproductiva, así como de prevención de enfermedades y embarazos adolescentes, específicamente en lo relacionado con el VIH, el uso del condón y la realización de la prueba de VIH. Por otra parte, la principal limitación de este estudio fue que el 
156

muestreo no aleatorio empleado no permite la generalización de los resultados en esta población. La validación de los instrumentos Barreras hacia la Prueba del VIH (Peralta et al., 2007) y Escala de Actitudes hacia el VIH-Sida (Espada et al., 2013) en población colombiana se presenta como una oportunidad de estudio para investigadores interesados en el tema. Por último, se recomienda que futuros trabajos continúen explorando, desde diferentes diseños metodológicos, los factores psicosociales asociados a la baja realización de la prueba diagnóstica del VIH.

\section{Referencias}

Arrivillaga, M., Hoyos, P. A., Tovar, L. M., Zapata, H., Varela, M. T., \& Correa, D. (2012). HIV Testing and Counseling in Colombia: Evidence from a National Health Survey and Recommendations for Public Policy. International Journal of STD \& AIDS, 23(11), 815-821. doi: 10.1258/ ijsa.2012.011468

Becky, L. W., Walsh, J., Raysam, S., Pathman, D. E., Adimora, A. A., \& Golin, C. E. (2015). What Makes Me Screen for HIV? Perceived Barriers and Facilitators to Conducting Recommended Routine HIV Testing among Primary Care Physicians in the Southeastern United States. Journal of the International Association of Providers of AIDS Care, 14(2), 127-135. doi: $10.1177 / 2325957414524025$

Begley, K., McLaws, M. L., Ross, M. W., \& Gold, J. (2008). Cognitive and Behavioral Correlates of Non-Adherence to HIV Anti-Retroviral Therapy: Theoretical and Practical Insight for Clinical Psychology and Health Psychology. Clinical Psychologist, 12(1), 9-17. doi: 10.1080/13284200802069043

Belza, M. J., Figueroa, C., Rosales, M. E., Ruiz, M., Vallejo, F., $\&$ de la Fuente, L. (2014). Low Knowledge and Anecdotal Use of Unauthorized Online HIV Self-Test Kits among Attendees at a Street-Based HIV Rapid Testing Programme in Spain. International Journal of Infectious Diseases, 25, 196-200. doi: 10.1016/j.ijid.2014.03.1379

Bilardi, J. E., Walker, S., Read, T., Prestage, G., Chen, M. Y., Guy, R., ... Fairley, C. K. (2013). Gay and Bisexual Men's Views on Rapid Self-Testing for HIV. AIDS and Behavior, 17(6), 2093-2099. doi: 10.1007/s10461-012-0395-7

Black, S., Wallace, M., Middelkoop, K., Robbertze, D., Bennie, T., Wood, R., \& Bekker, L. G. (2014). Improving HIV Testing Amongst Adolescents through an Integrated Youth Centre Rewards Program: Insights from South Africa. Children and Youth Services Review, 45(C), 98-105. doi: 10.1016/j.childyouth.2014.03.025

Bolsewicza, K., Vallelyb, A., Debattistad, J., Whittakere, A., \& Fitzgeralda, L. (2015). Factors Impacting HIV Testing: A Review - Perspectives from Australia, Canada, and the UK. AIDS Care, 27(5), 570-580. doi: $10.1080 / 09540121.2014 .986050$
Bradley, H., Tsui, A., Kidanu, A., \& Gillespie, D. (2011). Client Characteristics and HIV Risk Associated with Repeat HIV Testing among Women in Ethiopia. AIDS and Behavior, 15(4), 725-733. doi: 10.1007/s10461-010-9765-1

Brooks, R. A., Lee, S. J., Stover, G. N., \& Barkley, T. W. (2011). HIV testing perceived vulnerability and correlates of HIV sexual risk behaviors of Latino and African American young male gang members. International Journal of STD \& AIDS, 22(1), 19-24. doi: 10.1258/ijsa.2010.010178

Castel, A. D., Befus, M., Willis, S., Griffin, A., West, T., Hader, S., ... Greenberg, A. E. (2012). Use of the Community Viral Load as a Population-Based Biomarker of HIV Burden, Washington, DC. AIDS, 26(3), 345-353. doi: 10.1097/ QAD.0b013e32834de5fe

Cohen, J. (1988). Statistical Power Analysis for the Behavioral Sciences. Nueva York, Estados Unidos: Routledge.

Cooney, N., Hiransuthikul N., \& Lertmaharit, S. (2015). HIV in Young People: Characteristics and Predictors for Late Diagnosis of HIV. AIDS Care, 27(5), 561-569. doi: 10.1080/09540121.2014.986049

Dai, S., Liu, J., Fan, Y., Shan, G., Zhang, H., \& Li, M. (2015). Prevalence and Factors Associated with Late HIV Diagnosis. Journal of Medical Virology, 87(6), 970-977. doi: $10.1002 / j m v .24066$

Decker, M. R., Rodney, R., Chung, S., Jennings, J. M., Ellen, J. M., \& Sherman, S. G. (2015). HIV Testing among Youth in a High-Risk City: Prevalence, Predictors, and Gender Differences. AIDS Care, 27(5), 555-560. doi: 10.1080/09540121.2014.986048

Deblonde, J., De Koker, P., Hamers, F. F., Fontaine, J., Luchters, S., \& Temmerman, M. (2010). Barriers to HIV Testing in Europe: A Systematic Review. The European Journal of Public Health, 20(4), 422-432. doi: 10.1093/eurpub/ckp231

Encuesta Nacional de Demografía y Salud [ENDS]. (2015). Conocimiento de ITS y del VIH/Sida: formas de prevención. Recuperado de http://profamilia.org.co/docs/ENDS\%20 TOMO\%20II.pdf

Espada, J., Ballester, R., Huedo, T., Secades, R., Orgilés, M., \& Martínez, M. (2013). Development of a new instrument to assess AIDS-related attitudes among Spanish Youngsters. Anales de Psicología, 29(1), 83-89. doi: 10.6018/ analesps.29.1.132301

Espada, J. P., Escribano, S., Morales, A., \& Orgile, M. (2017). Two-Year Follow-Up of a Sexual Health Promotion Program for Spanish Adolescents. Evaluation \& The Health Professions, 40(4), 1-22. doi: 10.1177/0163278716652217

Espada, J. P., Escribano, S., Orgilés, M., Morales, A., \& Guillén, A. (2015). Sexual risk behaviors increasing among adolescents over time: comparison of two cohorts in Spain. AIDS Care, 27(6), 783-788. doi: 10.1080/09540121.2014.996516

Flowers, P., Knussen, C., Li, J., \& McDaid, L. (2013). Has Testing Been Normalized? An Analysis of Changes in Barriers to HIV Testing among Men Who Have Sex With Men between 
2000 and 2010 in Scotland, UK. HIV Medicine, 14(2), 9298. doi: 10.1111/j.1468-1293.2012.01041.x

Fuster, M. J., Molero, F., Nouvilas, E., Arazo, P., \& Dalmau, D. (2014). Determinants of Late Diagnosis of HIV Infection in Spain. Psychosocial Intervention, 23(3), 177-185. doi: 10.1016/j.psi.2014.07.011

García, P., Reyes, J. M., \& Caylà, J. A. (2012). Retraso diagnóstico de la infección por VIH. Revista Española de Sanidad Penitenciaria, 14(1), 28-35. Recuperado de http://scielo. isciii.es/scielo.php?script $=$ sci_arttext\&pid $=$ S1575-062020 $12000100005 \& \operatorname{lng}=$ es\&nrm=iso\&tlng=es

Hampanda, K., Ybarra, M., \& Bull, S. (2014). Perceptions of Health Care Services and HIV-Related Health-Seeking Behavior among Uganda Adolescents. AIDS Care, 26(10), 1-9. doi: 10.1080/09540121.2014.894612

Hernández, R., Fernández, C., \& Baptista, P. (2010). Definición del alcance de la investigación a realizar: exploratoria, descriptiva, correlacional o explicativa. En R. Hernández, C. Fernández y P. Baptista (eds.), Metodología de la investigación (pp. 76 - 88). México D. F.: Mc-Graw-Hill.

Hoyos, J., de la Fuente, L., Fernández, S., Gutiérrez, J., Rosales, M. E., \& de Olalla, P. G. (2012). La oferta de la prueba rápida del VIH en la calle dentro del ámbito universitario: ¿una estrategia prioritaria? Gaceta Sanitaria, 26(2), 131-137. doi: 10.1016/j.gaceta.2011.07.009

Hoyos, J., Fernández, S., de la Fuente, S., Sordo, L., Ruiz, M., Barrio, G., \& Belza, M. J. (2013). Never Tested For HIV in Latin-American Migrants and Spaniards: Prevalence and Perceived Barriers. Journal of the International AIDS Society, 16(1), 1-8. doi: 10.7448/IAS.16.1.18560

Hoyos, P. A., Sanabria, J. P., Orcasita, L. T., Valenzuela, A. L., González, M., \& González, T. (2019). Social representations associated with HIV/AIDS in Colombian university students. Saúde e Sociedade, 28(2), 227-238. doi: 10.1590/ S0104-12902019180586

Hyden, C., Allegrante, J. P., \& Cohall, A. T. (2014). HIV Testing Sites' Communication about Adolescent Confidentiality: Potential Barriers and Facilitators to Testing. Health Promotion Practice, 15(2), 173-180. doi: $10.1177 / 1524839913499347$

Infosida. (2016). Preguntas frecuentes sobre VIH. Recuperado de https://www.infosida.es/preguntas-frecuentes-vih

Janz, N. K., \& Becker, M. H. (1984). The Health Belief Model: A Decade Later. Health Education \& Behavior, 11(1), 1-47. doi: 10.1177/109019818401100101

Kaai, S., Bullock, S., Burchell, A. N., \& Major, C. (2012). Factors that Affect HIV Testing and Counseling Services among Heterosexuals in Canada and the United Kingdom: An Integrated Review. Patient Education and Counseling, 88(1), 4-15. doi: 10.1016/j.pec.2011.11.011

Kall, M. M., Smith, R. D., \& Delpech, V. C. (2012). Late HIV Diagnosis in Europe: A Call for Increased Testing and Awareness among General Practitioners. European Journal of General Practice, 18(3), 181-186. doi:10.3109/1381478 8.2012.685069

Kurth, A. E., Lally, M. A., Choko, A. T., Inwani, I. W., \& Fortenberry, J. D. (2015). HIV Testing and Linkage to Services for Youth. Journal of the International AIDS Society, 18(Sup. 1), 23-28. doi: 10.7448/IAS.18.2.19433

Lopera, M. M., Martínez, J., \& Ray, T. (2011). Acceso de las personas con VIH al sistema de salud colombiano y sus costos relacionados desde una perspectiva individual y familiar, Bogotá, 2010. Revista Gerencia y Políticas de Salud, 10(20), 81-96. Recuperado de http://www.scielo.org.co/ pdf/rgps/v10n20/v10n20a06.pdf

Ministerio de Salud y Protección Social. (1997). Cuestionario Confidencial sobre Vida Sexual Activa. Bogotá, Colombia: Ministerio de Salud de Colombia.

Ministerio de Salud y Protección Social. (2011). Resolución 2338 de 2013. Recuperado de http://www.alcaldiabogota. gov.co/sisjur/normas/Norma1.jsp?i=53670

Ministerio de Salud y Protección Social. (2015). Guía para el manejo del VIH/Sida. Basado en la evidencia. Recuperado de https://www.minsalud.gov.co/Documentos $\% 20 \mathrm{y} \% 20$ Publicaciones/GUIA\%20PARA\%20EL\%20MANEJO\%20 DE\%20VIH\%20SIDA.pdf

Morales, A., Espada, J. P., \& Orgilés, M. (2016). Barreras hacia la prueba de detección del VIH en adolescentes en España. Psychosocial Intervention, 25(3), 135-141. doi: 10.1016/j. psi.2016.06.002

Newman, C., Kidd, M., Kippax, S., Reynolds, R., Canavan, P., \& de Wit, J. (2013). Engaging non HIV Specialist General Practitioners with New Priorities in HIV Prevention and Treatment: Qualitative Insights from Those Working in the Field. Sexual Health, 10(3), 193-198. doi: 10.1071/sh12157

Organización Mundial de la Salud [OMS].(2012). Orientaciones clínicas aplicables a lo largo de todo el proceso asistencial: diagnóstico del VIH y uso de ARV con fines preventivos. Recuperado de http://www.who.int/hiv/pub/guidelines/ arv2013/clinical/arv2013_chapter05_es.pdf

Peralta, L., Deeds, B. G., Hipszer, S., \& Ghalib, K. (2007). Barriers and Facilitators to Adolescent HIV Testing. AIDS Patient Care and STDs, 21(6), 400-408. doi: 10.1089/ apc.2006.0112

Profamilia. (2017). Preguntas y respuestas sobre VIH. Recuperado de https://profamilia.org.co/ preguntas-y-respuestas/vihsida/?id=14

Schnall, R., Rojas, M., \& Travers, J. (2015). Understanding HIV Testing Behaviors of Minority Adolescents: A Health Behavior Model Analysis. Journal of the Association of Nurses in Aids Care, 26(3), 246-258. doi: 10.1016/j. jana.2014.08.005

Sevilla, T. M., Sanabria, J. P., Orcasita, L. T., \& Palma, D. M. (2016). Consistencies and Discrepancies in Communication between Parents and Teenage Children about Sexuality. Paidéia (Ribeirão Preto), 26(64), 139-147. doi: $10.1590 / 1982-43272664201601$ 